\title{
Spiradenoma of the breast and review of cases over 76-years
}

\author{
Mary K. Dick*1, Apporva Sharma ${ }^{1}$, Poonam Sharma ${ }^{2}$ \\ ${ }^{1}$ Creighton School of Medicine, Omaha, Nebraska, USA \\ ${ }^{2}$ Pathology Department, CHI Health Creighton University Medical Center Bergan Mercy, Omaha, Nebraska, USA
}

Received: October 8, 2019

DOI: $10.5430 /$ crcp.v6n1p38
Accepted: October 22, 2019

Online Published: November 23, 2019

\begin{abstract}
Spiradenomas stem from eccrine and apocrine glands to form benign cutaneous tumors. When arising on the breast, nipple, and areola region, spiradenomas can be difficult to differentiate from more worrisome breast masses. This is particularly true in patients with a prior history of breast cancer. These tumors can be mimicked by a wide range of adnexal growths and play a role in syndromic conditions. We present a case of a 56-year-old female with a 20-year enlarging left lower inner quadrant breast mass reviewed on mammography which was later excised and histopathologically diagnosed as a spiradenoma. Spiradenomas can be challenging to diagnose as they can present with similar findings on inexpensive and non-invasive testing such as ultrasound and mammogram. While a biopsy is the definitive tool for diagnosis, this is contraindicated in some spiradenoma mimickers such as epidermal cysts complicating diagnosis. To our knowledge there are only six case reports of breast spiradenomas. We have reviewed the histopathology of this case and summarized all cases of breast spiradenomas and their malignant counterpart spiradenocarcinomas available in the literature compiling their presentations and imaging findings.
\end{abstract}

Key Words: Spiradenoma, Spiradenocarcinoma, Breast mass, Brooke-Spiegler syndrome

\section{INTRODUCTION}

Spiradenomas were initially described in the literature in 1956 as benign skin lesions stemming from eccrine or apocrine sweat glands. Classically, lesions are solitary, firm, painful, measure under two centimeters, and can be located within the dermis on the head, neck, trunk, and extremities. Most frequently, spiradenomas are encountered on the head and neck with only rare occurrences on the breast. ${ }^{[1,2]}$ Development is most common between the ages of 20-40 years-old in both males and females (1:1 ratio) without racial preference. ${ }^{[1,3]}$ Lesions often grow for 20-30 years before necessitating medical attention. Only six cases of benign breast spiradenomas have been previously reported in the literature. Additionally, their malignant counterpart, spiradenocarcino- mas, are even more infrequently found in this location. The characteristics of both tumors in all reported cases in the literature over the past 76 years are displayed in Table $1 .{ }^{[1-19]}$

\section{CASe PResentation}

A 56-year-old female presented for inspection of a $3 \mathrm{~cm}$ dermal lesion on the lower inner quadrant of the left breast that has been enlarging over twenty years. Upon initial examination, the lesion was presumed to be a sebaceous cyst or a lipoma. The patient's most recent mammogram showed scattered fibroglandular density within the breast and highlighted the superficial nature of this peripheral skin lesion. She was considered a high cancer risk as family members were previously diagnosed with cancer of the breast, pancreas,

*Correspondence: Mary K. Dick; Email: MaryDick@creighton.edu; Address: 7500 Mercy Road, Omaha, Nebraska, 68124, USA. 
prostate, bone, lung, colon, esophagus and lymphatic system. Additionally, the patient underwent analysis of BRCA1 and BRCA2 gene mutations with negative results. The lesion was removed with an elliptical excision without any additional margins. Resulting histopathology returned with a diagnosis of spiradenoma.

The histologic specimen shows two types of tumor cells within well circumscribed subcutaneous dermal nodules. Small basaloid cells are located peripherally surrounding large pale cells containing centrally situated eosinophilic cy- toplasm. Lymphocyte infiltration, abundant fine vasculature, and lack of significant atypia or mitotic activity are additionally identified within the specimen. Tubule formation is appreciated within central tumor cells with surrounding ducts are highlighted by the positive stains of the following: Periodic Acid Schiff cytokine 5/6 immunohistochemical stain (IHC) carcinoembryonic antigen (CEA) and epithelial membrane antigen IHC, P63 IHC, gross cystic disease fluid protein $15 \mathrm{IHC}$, and endoplasmic reticulum IHC (see Figures $1-3)$.

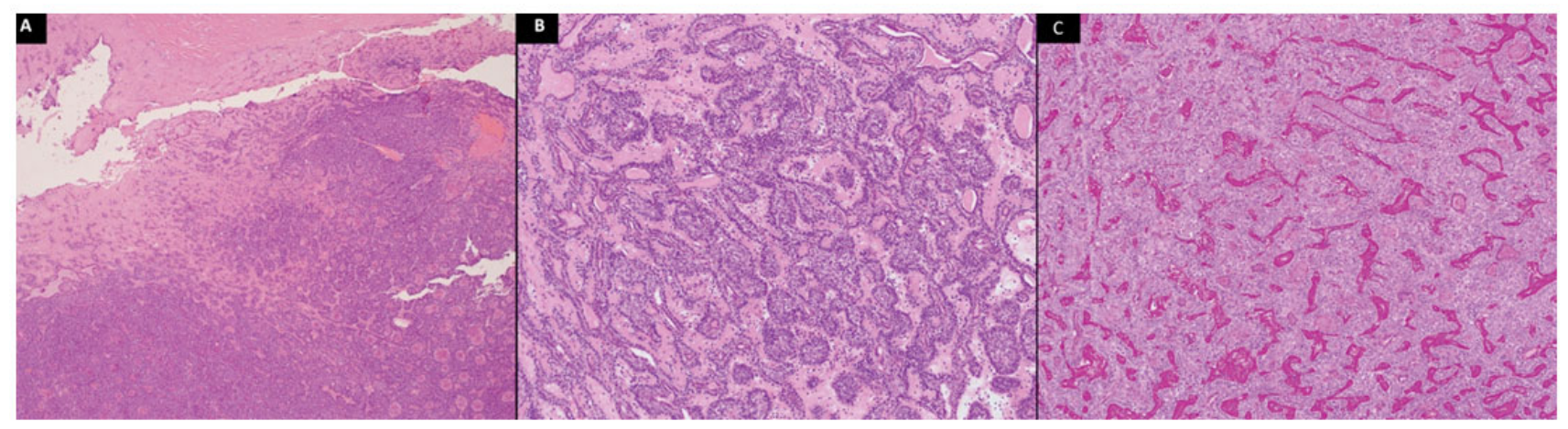

Figure 1. Histopathologic findings of spiradenoma hematoxylin and eosin stain and Periodic acid-Schiff stain. Image (A) shows a spiradenoma with H\&E stain, 4X. At low power, the tumor appears as a solid neoplasm composed of basaloid cells. (B) H\&E 10x. The nodules are encapsulated by a thick fibrous capsule. (C) Spiradenoma, periodic acid-Schiff (PAS) stain, 10X. PAS-positive hyaline material present within the tumor is characteristic of spiradenoma.

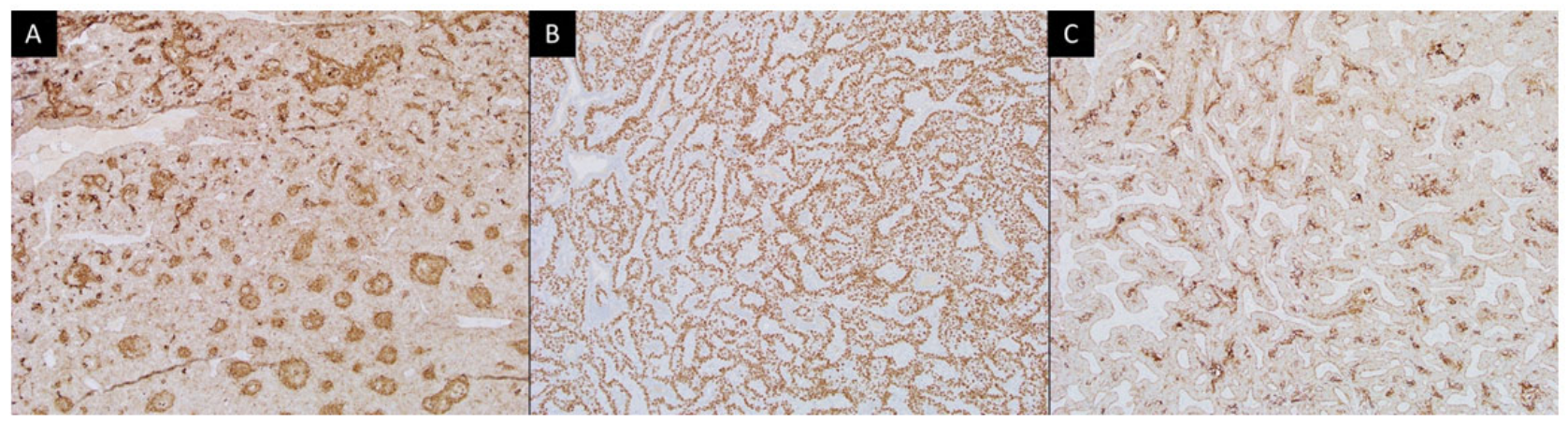

Figure 2. Histopathologic findings of spiradenoma cytokeratin (CK) 5/6 immunohistochemical (IHC) stain, P63 IHC, and CK 8/18 IHC stains. Image (A) shows a spiradenoma with cytokeratin (CK) 5/6 immunohistochemical (IHC) stain, 10X. Immunohistochemical staining of the tumors revealed positive immunoreactions for CK 5/CK6. Central darker and peripheral weaker staining cells are present. (B) Tumor protein P63 IHC, 10X (first area). Two distinct populations of neoplastic cells can be seen in the tumor with p63 immunohistochemical stain showing nuclear positivity in the outer cells and no staining in inner ductal cells. (C) CK 8/18 IHC, 10X Positive reaction of tumor with low molecular weight CK 8/18 immunohistochemical stain. Dimorphic population with central darker staining cells forming the ductal structures and peripheral weaker staining cell are seen.

Immunohistochemical staining of the tumors revealed positive immunoreactions for CK 5/CK6. Central darker and peripheral weaker staining cells are present. (B) Tumor pro- tein P63 IHC, 10X (first area). Two distinct populations of neoplastic cells can be seen in the tumor with p63 immunohistochemical stain showing nuclear positivity in the outer 
cells and no staining in inner ductal cells. (C) CK 8/18 IHC, tion with central darker staining cells forming the ductal 10X Positive reaction of tumor with low molecular weight structures and peripheral weaker staining cell are seen.

CK 8/18 immunohistochemical stain. Dimorphic popula-

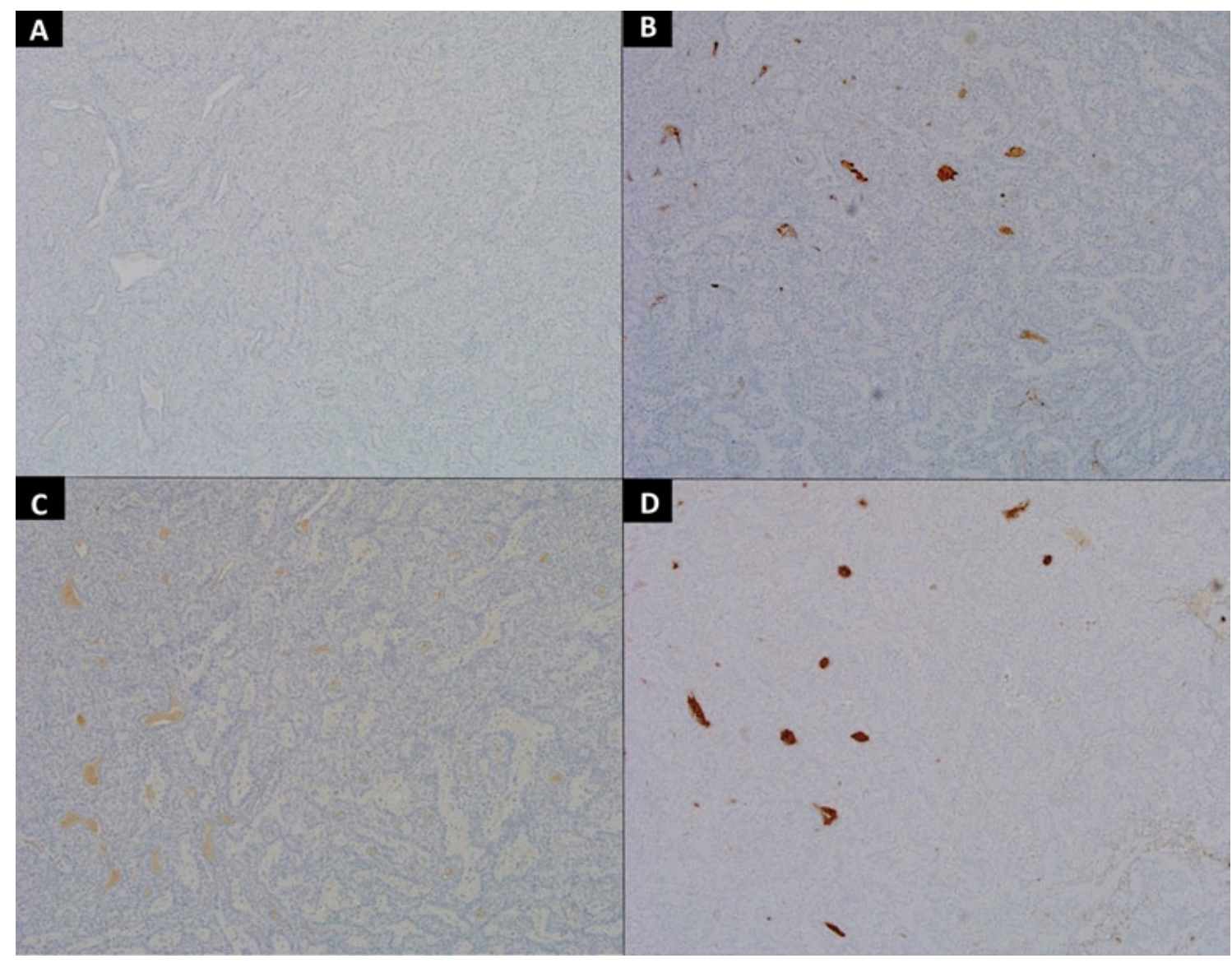

Figure 3. Histopathologic findings of spiradenoma endoplasmic reticulum IHC, Epithelial membrane antigen IHC, Gross cystic disease fluid protein 15, and carcinoembryonic antigen stains. Image (A) shows estrogen receptor IHC, 10X. Tumor cells show no nuclear staining for estrogen receptor (ER) immunohistochemical stain. ER stain is usually positive in breast tumors. (B) Epithelial membrane antigen (EMA) IHC, 10X. Staining with EMA immunohistochemical stain shows luminal positivity highlighting the ductal lumina. (C) Gross cystic disease fluid protein 15 (GCDFP15) IHC, 10X. Tumor cells show no staining for GCDFP15 IHC. GCDFP15 may be expressed in luminal cells of spiradenoma as well as breast tumor. (D) Carcinoembryonic antigen (CEA) IHC, 10X. Staining with CEA immunohistochemical stain shows luminal positivity highlighting the ductal lumina.

\section{Discussion}

The differential diagnoses to consider alongside spiradenoma are broad. Spiradenocarcinoma, angioma, angioleiomyoma, neuroma, cyst, cylindroma and a collection of other painful skin tumors, remembered by the mnemonic created in 1993 "LEND AN EGG," encompassing leiomyoma, neuroma, dermatofibroma, angiolipoma, neurilemmoma, endometrioma, glomus tumor, and granular cell tumor are all reasonable to consider. $^{[4]}$ Spiradenomas can appear alongside cylindromas as both tumors originate from the eccrine gland. Jukic et al. published the only case in the literature of a breast lesion containing features of both spiradenoma and cylin- droma within the same tumor. ${ }^{[5]}$ The co-occurrence of these two tumors separately is frequently seen in Brooke-Spiegler syndrome, a condition where various adnexal neoplasms develop due to a CYLD tumor suppressor gene mutation located on 16q12-q13 normally interacting with the anti-apoptotic nuclear factor kappa B (NF-kB) pathway. ${ }^{[6,7]}$ While this pathway has been identified for this syndromic condition the exact pathogenesis of solitary spiradenomas has not yet been elucidated.

Within the 14 total cases of spiradenomas and spiradenocarcinomas reported, all patients were female with lesions (when specified) located in the upper outer quadrant of the breast or 
within the nipple region. Of the 14 cases, $50 \%$ were benign. The average age of the patient was 54.7 years old. Lesions were present from 3 months to 50 years. Mammography addressed the lesions in 7 cases. Ultrasound findings showed hypoechoic structures in 2 of the cases. Immediate excision occurred in 5 cases. Lumpectomy was opted for in 1 case.

Malignant transformation from benign lesions occurs more frequently compared to de novo spiradenocarcinoma growth. Transformation should be suspected with rapid growth, color change, and/or ulceration. ${ }^{[5]}$ The time from a benign to malignant lesion is variable, but a range of 20-30 years appears to be an agreed upon average in the literature. ${ }^{[8]}$ Once malignant, spiradenocarcinomas have a metastasis rate of $50 \%$ spreading most commonly to the lymph nodes, bone, lungs, brain, and liver. ${ }^{[1,9]}$

Adding to the difficulty of spiradenoma differentiation, a biopsy is required for diagnosis but can be recommended against in cases like epidermal cysts as nodule penetration can lead to leakage of cyst contents generating inflammation. ${ }^{[10]}$ Imaging has been utilized as diagnostic aid. There is currently a paucity of image reporting on spiradenoma of the breast in part due to the uncommon presentation. If imaging is desired to differentiate a cutaneous origin from a breast mass, ultrasound is cost-effective and can additionally confirm a cystic or solid nodule structure. Spiradenomas and epidermal inclusion cysts appear similar on ultrasound and mammography but have differentiating features on magnetic resonance imaging. ${ }^{[1]}$ Both in ultrasound and mammograms, the lesions appear as a well-defined rounded mass with varying differences in density dependent on levels of debris within the epidermal inclusion cysts. There is only one report of MRI findings of spiradenomas of the breast by Lee et. al.. In this report, Spiradenomas T1-weighted images displayed low signal intensity, T2-weighted images displayed medium to low signal intensity, and turbo inversion recovery magnitude (TIRM) images had high signal intensity. Compared to epidermal inclusion cysts, T1-weighted images show high signal intensity. T2-weighted images are variable as the intensity of signal appearance is dependent on cysts content and structure. ${ }^{[10]}$ Confusion of adnexal tumors from breast cancer is in part due to difficulty localizing the lesion origin on a screening mammogram due to lower spatial resolution when compared to ultrasound. Spiradenomas are classically seen within the superficial and deep dermis. A lesion that is strictly within the dermis is typically considered benign on ultrasound. Superficial breast cancers can appear in the hypodermis and dermal-hypodermal junction. Skilled operators and close attention with identification of the tumor location within specific skin layers is needed to make the decision for further investigation. ${ }^{[20]}$

Spiradenoma diagnosis is ultimately based on histopathologic findings from excisional, fine needle aspiration, or core biopsy specimens. These eccrine tumors classically consist of well-defined lobules surrounded by an edematous fibrovascular stroma embedded with epithelial cords, tubules, and hyaline matter. Classically, composing the tumor are two separate epithelial cell populations appreciated on cytology. Centrally located are large cells containing pale nuclei with peripheral surrounding basaloid cells that are smaller in size with compressed and crowded nuclei. ${ }^{[5]}$ Permeating the surrounding stroma are small unmyelinated axons thought to be the source of the pain elicited by the tumor. ${ }^{[11]}$

\section{CONFLicts OF INTEREST Disclosure}

The authors declare that they have no competing interest.

\section{REFERENCES}

[1] Gomez A, Moratalla CN, Ferrer F, et al. Malignant eccrine breast spiradenoma. A case report and literature review. Int J Surg Case Rep. 2015; 15: 81-84. PMid:26318134. https://doi.org/10.1016/ j.ijscr.2015.08.017

[2] Bosch M, Boon M. Fine-needle cytology of an eccrine spiradenoma of the breast: Diagnosis made by a holistic approach. Diagn Cytopathol. 1992; 8(4): 366-368. PMid:1322262. https://doi.org/ $10.1002 / \mathrm{dc} .2840080412$

[3] Benedict M, Ozerdem U. Case Report Eccrine spiradenoma arising from the breast skin. Case Rep Pathol. 2015; 2015: 615158. PMid:26236527. https://doi .org/10.1155/2015/615158

[4] Naversen D, Trask D, Watson F, et al. Painful tumors of the skin: "lend an egg". J Am Acad Dermatol. 1993; 2(Pt 2): 298-300. https://doi.org/10.1016/0190-9622 (93) 70039-V

[5] Jukic D, Drogowski L, Davie J. Carcinoma ex Spirade-

Published by Sciedu Press
noma/Cylindroma confirmed by immunohistochemical and molecular loss-of-heterozygosity profiling. Am J Dermatopathol. 2009; 31(7). PMid:19684510. https://doi.org/10.1097/DAD.0b013e3181 $\mathrm{a} 6 \mathrm{~b} 6 \mathrm{f} 4$

[6] Vanecek T, Halbhuber Z, Kacerovska D, et al. Large germline deletions of the CYLD gene in patients with Brooke-Spiegler syndrome and multiple familial trichoepithelioma. Am J Dermatopathol. 2014; 36(11): 868-874. PMid:25347032. https://doi.org/10.1097/ DAD. 0000000000000068

[7] [7] Blake P, Torro J. Update of cylindromatosis gene (CYLD) mutations in brooke-spiegler syndrome: Novel insights into the role of deubiquitination in cell signaling. Hum Mutat. 2009; 30(7): 1025-1036. PMid:19462465. https://doi .org/10.1002/humu. 21024

[8] Ribeiro-Silva A, Shaletich C, Careta R, et al. Spiradenocarcinoma of the breast arising in a long-standing spiradenoma. Ann Diagn Pathol 2004; 8(3): 162-166. PMid:15185264. https://doi.org/10.101 
6/j.anndiagpath.2004.03.007

[9] Kanwaljeet S, Chatterjee T. Eccrine spiradenoma: A rare adnexal tumor. Indian J Cancer. 2017; 54(4): 695-696. PMid:30082561. https://doi.org/10.4103/ijc.IJC_301_17

[10] Lee H, Park S, Choi H, et al. Eccrine spiradenoma arising in the breast misdiagnosed as an epidermal inclusion cyst. Korean J Radiol. 2011; 12(2): 256-60. PMid:21430945. https ://doi.org/10.334 8/kjr.2011.12.2.256

[11] Park H, Im S, Shin D, et al. Painful eccrine spiradenoma containing nerve fibers: A case report. Dermatology. 2012; 224(4): 301-306. PMid:22777362. https://doi.org/10.1159/000339768

[12] Gingrich A, Fung M, Konia T, et al. Locally advanced spiroadenocarcinoma in the regional axilla of a breast cancer patient: Hallmarks of definitive diagnosis and management. Rare Tumors. 2015; 7(3): 5912 PMid:26500731. https://doi.org/10.4081/rt.2015.5912

[13] Son J, Choi Y, Cho Y, et al. A case of eccrine spiradenoma: A rarely seen soft tissue tumor on the extensor surface of arm. Ann Dermatol. 2017; 29(4): 519-522. PMid:28761313. https://doi.org/10.5 021/ad.2017.29.4.519

[14] Draheim J, Neubecker R, Sprinz H. An unusual tumor of the breast resembling eccrine spiradenoma. Am J Clin Pathol. 1959; 31(6): 511-516. PMid:13661107. https://doi.org/10.1093/ajcp/3 1.6 .511
[15] Metovic J, Gallino C, Zanon E, et al. Eccrine spiradenoma of the nipple: Case report, differential diagnosis and literature review. Histol Histopathol. 2019; 18094.

[16] Panico L, D'Antonio A, Chiacchio R, et al. An unusual, recurring breast tumor With Features of eccrine Spiradenoma A case report. Am J Clin Pathol. 1996; 106(5): 665-669. PMid:8929479. https://doi.org/10.1093/ajcp/106.5.665

[17] Saboorian M, Kenny M, Ashfaq R, et al. Carcinosarcoma arising in eccrine spiradenoma of the breast. report of a case and review of the literature. Arch Pathol Lab Med. 1996; 120(5): 501-504.

[18] Tanaka Y, Bhunchet E, Shibata T. A case of malignant eccrine spiradenoma metastatic to intramammary lymph node. 2008; 15(2): 175-180. PMid:18270794. https : //doi .org/10.1007/s12282 $-007-0023-y$

[19] Thomas B, Duwel V, Proot L, et al. An uncommon breast tumour: The malignant eccrine spiradenoma. A case report. Acta Chir Belg. 1993; 93(6): 295-298.

[20] Giess CS, Raza S, Birdwell RL. Distinguishing Breast Skin Lesions from Superficial Breast Parenchymal Lesions: Diagnostic Criteria, Imaging Characteristics, and Pitfalls. RadioGraphics. 2011; 31(7): 1959-1972. PMid:22084181. https ://doi.org/10.1148/rg.3 17115116 